\title{
Spectrochemical Micro-Analysis of Biological Tissues Using Raman and FT-IR Microspectroscopy with Metalographic Polishing
}

\author{
V. L. St. Jeor*, D. L. Elmore*, C. A. Lendon*, S. A. Smith*, C. L. Leverette** \\ * Cargill Scientific Resources, P.O. Box 3000, Cordova, TN 38088 \\ ** University of South Carolina Aiken, Department of Chemistry and Physics, Aiken, South \\ Carolina 29801-6399
}

Raman and Fourier transform infrared (FT-IR) microspectroscopies are powerful tools for investigating chemical structure on a micro-scale. Unfortunately, data quality is often compromised by sampling anomalies associated with optically rough surfaces. Chemical alteration during sample preparation and insufficient morphological preservation also contribute to problematic results. These problems are commonly encountered in the study of food, agricultural and biological specimens. In our lab, a method was needed that would permit sample interrogation by Raman and FT-IR microspectroscopies, while preserving structure integrity of an entire exposed botanical specimen. Although reasonable sample preparation methods exist for light and electron microscopic visualization of ultra-fine structure in tissue, many of these methods are designed to preserve only small regions of the tissue - often too small for spectrochemical analysis. And in our specific case, traditional methods do not sufficiently preserve structure throughout the entire kernel, nor its cross-section.

Methodology has been developed in the metalographic polishing industry that can produce extremely smooth, flat surfaces for many bulk-type materials. Interestingly, this methodology is rarely exploited in the field of biology (if at all). The idea of mechanically polishing a biological sample in preparation for spectrochemical analyses, may at first seem inconsistent with the goal of tissue preservation. However, in this paper we demonstrate that metalographic polishing may be used to prepare samples for Raman and FT-IR microspectroscopic analyses. The quality of tissue preservation is demonstrated with both light and scanning electron microscopy. Lack of crosscontamination between tissue types within the same biological sample is demonstrated by Raman measurements of different components separated by only a few micrometers. No measurable cross-contamination from neighboring components (such as oil, starch, protein, embedding resin, etc.) was identified.

Polishing impacts tissues differently depending upon how tightly neighboring tissues are bound and how well they resist abrasion. Additionally, for all materials, polishing lubricants are necessary for achieving good results. Possible lubricants may include water, alcohol, and biological fixatives. In one application, water leaves the native oil intact in a corn kernel, while absolute ethyl alcohol effectively extracts the oil. The extraction allows informative Raman spectra to be obtained that are not dominated by oil peaks. A biological fixative can be used to preserve fine structural details. Other applications will be presented and discussed. 

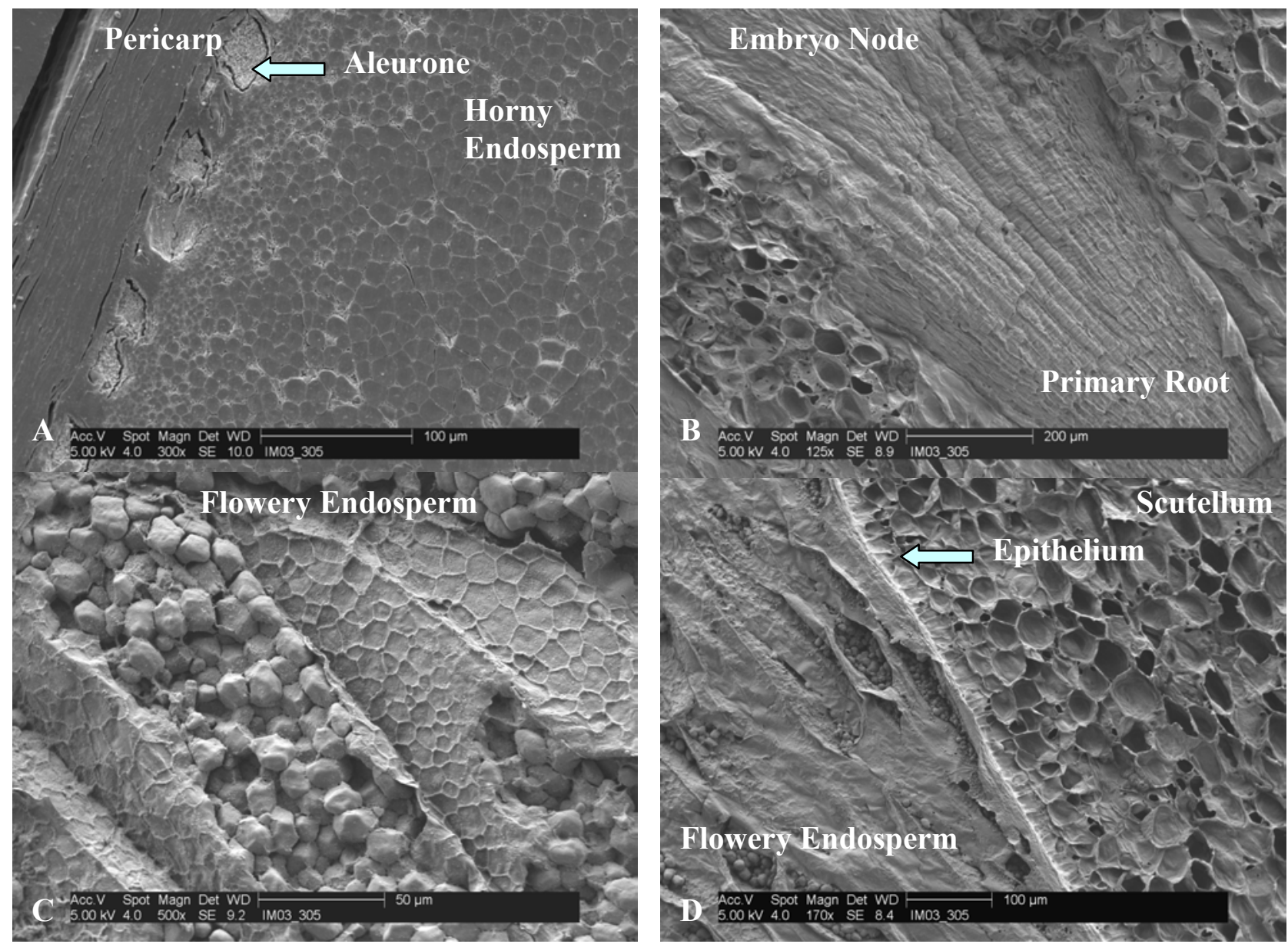

Fig.1a-d. Various SEM images of polished corn kernel tissue showing morphological preservation.
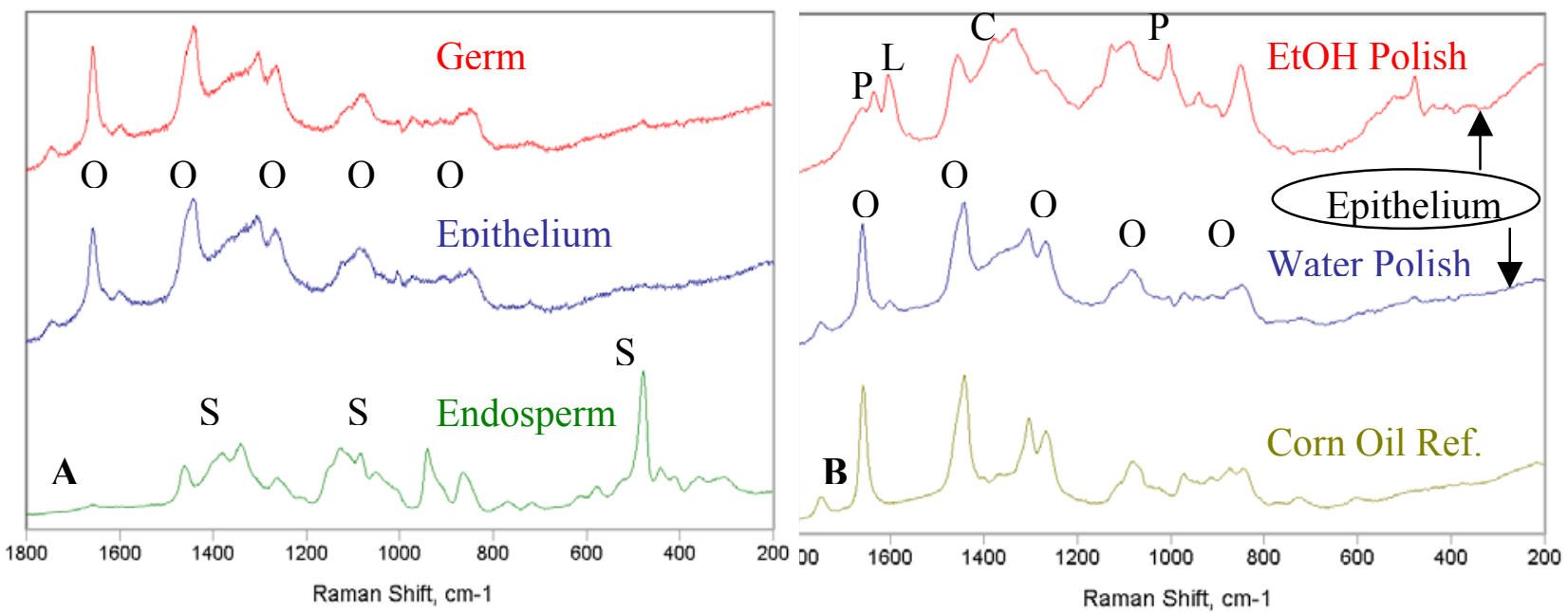

Fig.2a-b. Raman spectra indicating various chemical components in the corn kernel. C-

Carbohydrate, L - Lignin, O - Oil, P - Protein, S - Starch. Figure 2a shows spectra obtained for various tissues, using a water-based lubricant. No cross-contamination was identified between tissues separated by as little as a few micrometers. Figure $2 b$ shows the Epithelial tissue polished with either EtOH (top) or water (middle). The alcohol extracts a large amount of oil and allows other components to be readily identified. 